\title{
Duodenal juice stimulates oesophageal stem cells to induce Barrett's oesophagus and oesophageal adenocarcinoma in rats
}

\author{
TOMOHARU MIYASHITA ${ }^{1}$, TETSUO OHTA ${ }^{1}$, TAKASHI FUJIMURA ${ }^{1}$, ITASU NINOMIYA ${ }^{1}$, \\ SACHIO FUSHIDA ${ }^{1}$, TAKANORI HATTORI ${ }^{2}$ and KOICHI MIWA ${ }^{1}$ \\ ${ }^{1}$ Department of Gastroenterological Surgery, Division of Cancer Medicine, Graduate School of Medicine, \\ Kanazawa University, 13-1 Takaramachi Kanazawa, Ishikawa 920-8641; ${ }^{2}$ Department of Pathology, \\ Shiga University of Medical Science, Seta-tsukinowa-cho, Ohtsu, Shiga 520-2192, Japan
}

Received October 26, 2005; Accepted December 19, 2005

\begin{abstract}
The present study was performed to examine the sequential process of the development of Barrett's oesophagus (BE) and oesophageal adenocarcinoma (ADC) induced by duodeno-oesophageal reflux (DER) in rats. Total gastrectomy was performed in male Wistar rats weighing approximately $250 \mathrm{~g}$ followed by reconstruction with oesophago-jejunostomy, which causes unavoidable DER without exposure to exogenous carcinogens. Animals were selected at random and sacrificed every 10 weeks after surgery until 50 weeks. Severe squamous oesophagitis with erosion, regenerative thickening (RT), and basal cell hyperplasia $(\mathrm{BCH})$ were observed on the 10th week after surgery. On the 20th week, glandular structures that stained positively with Galactose oxidase-Schiff (foveolar metaplasia) were observed in the basal layer of the oesophageal squamous epithelium. On the 30th week, the glands developed and formed cysts that stained positively with concanavalin A (pyloric glandular metaplasia) and/or high-iron diamine and Alcian blue (intestinal metaplasia). From the 40th week after surgery, ADC cells surrounded by columnar-lined epithelium were found. Persistent stimulation with DER can alter the stem cells in the squamous epithelial basal layer leading to the formation of columnar-lined cells and subsequent ADC. Foveolar metaplasia was observed as part of the sequence of events leading to the development of columnar-lined epithelium (CLE), followed by the appearance of pyloric glandular metaplasia and intestinal metaplasia, completing the histogenesis of BE.
\end{abstract}

Correspondence to: Dr Tomoharu Miyashita, Department of Gastroenterological Surgery, Division of Cancer Medicine, Graduate School of Medicine, Kanazawa University, 13-1 Takaramachi Kanazawa, Ishikawa 920-8641, Japan

E-mail: tomo9321@yahoo.co.jp

Key words: Barrett's oesophagus, oesophageal adenocarcinoma, histogenesis, duodeno-oesophageal reflux, oesophageal stem cell

\section{Introduction}

Barrett's oesophagus (BE) is defined as replacement of the original squamous epithelial lining of the oesophagus with columnar-lined epithelium (CLE). BE is associated with a risk of malignant transformation to oesophageal adenocarcinoma (ADC) $(1,2)$. The most common cause of BE has been suggested to be mucosal damage caused by oesophageal reflux of gastric and/or duodenal contents (3-5). Several reports have indicated that duodenal contents reflux play an important role in the pathogenesis of oesophageal mucosal injury in animal models (6-9).

However, the mechanisms by which BE and ADC arise are obscure. Surgically induced defects in the oesophageal mucosa are normally regenerated with squamous epithelium. Mucosal defects occurring concomitantly with reflux can be repaired with CLE. These CLE may originate from creeping substitution of the junctional glandular epithelium into the defective oesophageal squamous mucosa (10), or may be derived from the oesophageal gland (11) or from squamous stem cells.

The present study was performed to investigate the sequential mucosal changes in BE and ADC induced by duodeno-oesophageal reflux (DER) without exogenous carcinogens, and to elucidate the histogenesis of squamous epithelium replacement using immunohistochemical and mucin histochemical analysis.

\section{Materials and methods}

Experimental animals. Male Wistar rats weighing approximately $250 \mathrm{~g}$ were used. Animals were housed three to a cage, and maintained at a constant room temperature of $22 \pm 3^{\circ} \mathrm{C}$ and $55 \pm 5 \%$ humidity with a 12 -h light-dark cycle. They were fed standard solid chow CRF-1 (Charles River, Japan) and tap water ad libitum.

Surgical procedures. After a 24-h fast, an upper abdominal incision was made under diethyl-ether inhalation anaesthesia. The glandular stomach and forestomach were removed, and the duodenal stump was then closed with sutures. The oesophageal stump was anastomosed end-to-side to the jejunum 


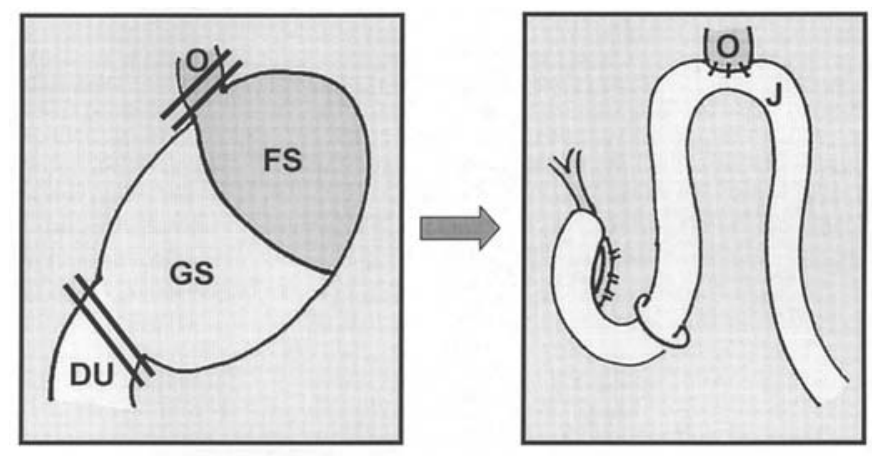

Figure 1. Diagram of surgical procedure. O, oesophagus; FS, forestomach; GS, glandular stomach; DU, duodenum; J, jejunum. In duodeno-oesophageal reflux, after total gastrectomy with closure of the duodenal stump, the oesophageal stump was then anastomosed end-to-side to the jejunum approximately $4 \mathrm{~cm}$ distal to Treiz's ligament, which induced reflux of the duodenal contents into the oesophagus.

approximately $4 \mathrm{~cm}$ distal to Treitz's ligament to induce reflux of duodenal contents into the oesophagus (Fig. 1).

Intestinal anastomosis was carried out with interrupted stitches of all layers in a line using 7-0 atraumatic silk-braided sutures. After surgery, the animals were allowed to drink immediately but fasting was continued for 2 days. Animals selected at random were sacrificed every 10 weeks after surgery until 50 weeks, which duration was based on the results reported previously $(7,8)$.

Specimen extraction. The animals were sacrificed by diethylether inhalation, after which the abdomen was opened. A ligature was placed around the afferent and efferent jejunal loop near the oesophago-jejunostoma, and the oesophagus was ligated at the level of the thyroid cartilage through a thoracotomy. The oesophagus and the anastomosed jejunum were then removed.

Histological examination. Excised organs were washed with $10 \%$ formalin, spread and pinned on a cork plate with the mucosal side up. After fixing with $10 \%$ formalin solution for at least $24 \mathrm{~h}$, the oesophagus was cut into slices at 3-mm intervals along the longitudinal axis, embedded in paraffin, and sections of each block $5 \mu \mathrm{m}$ thick were prepared for pathological analysis with haematoxylin-eosin (HE) staining.

Mucin histochemical staining. Histochemical staining was performed to identify various cells types positive for the following mucins in the epithelium of various lesions: galactose oxidase-Schiff (GOS) staining was performed according to the method reported previously to identify gastric surface mucous and foveolar epithelial cells (11); paradoxical concanavalin A (Con A) staining was performed according to the method of Katsuyama and Spicer (12) for class III mucin, which is found in the gastric mucous neck and pyloric gland cells; and highiron diamine and Alcian blue (HID-AB) staining, which are characteristically seen in the intestine and are indicative of sulphomucin and sialomucin (13).

Immunohistochemistry. We performed immunohistochemical staining for proliferating cell nuclear antigen (PCNA) to investigate the cell kinetic zone of CLE and ADC. PCNA staining (Cambridge, MA, USA) was performed using a three-step indirect immunoperoxidase method (streptavidinbiotin-peroxidase complex) according to the method of Ohta (14) with slight modifications. Slides were counterstained lightly with HE.

Definition of pathological findings. Oesophageal histological findings were classified into the following 5 categories.

Regenerative thickening: an oesophageal epithelial layer more than double the normal thickness, together with acanthosis, abnormal extension of papillae toward the mucosal surface, and parakeratosis. No disturbance of the stratified structure of the epithelium.

Basal-cell hyperplasia: thickening of the basal layer of the squamous epithelium resulting in its occupation of $>15 \%$ of the epithelial layer. Intramural cysts may also be present. The stratified structure of the squamous epithelium is preserved.

Erosion: defects of the epithelium with inflammatory cell infiltration.

Barrett's oesophagus: replacement of oesophageal squamous epithelium with columnar-lined epithelium comprised of gastric and/or intestinal cells.

Carcinoma: carcinoma was defined as epithelial growth with cellular and structural atypism, invading into the submucosal layer. ADC consists of dysplastic glandular cell growth with both atypia and invasiveness and has two types of histology: tubular or papillary adenocarcinoma, and mucinous adenocarcinoma. Squamous cell carcinoma (SCC) is characterized by marked cellular and structural atypism. This carcinoma may be divided into well-differentiated and poorly differentiated types according to the presence or absence of cancer pearls, respectively. Adenosquamous carcinoma (ASC) is composed of two components, ADC and SCC.

\section{Results}

Sixty-three animals survived after surgery and were used in the present study.

Macroscopic findings. On the 20th and 30th week after surgery, the lower oesophageal wall was wide and thickened in all animals. The mucosa showed longitudinal zonal changes: it had a glistening surface giving it a cobblestone appearance in the lower portion, uneven surface with erosion in the middle portion, normal mucosa in the upper portion, and lacked a sharp horizontal demarcation line between the oesophageal and jejunal epithelia (Fig. 2A and B). After the 40th week, large polypoid tumours were observed in the lower oesophagus (Fig. 2C), one of which was found to have invaded to the liver (Fig. 2D).

Microscopic findings. All animals showed an oesophageal inflammatory response consisting of infiltration of small round cells, mainly lymphocytes, in the 10th week after surgery, and this became marked after the 20th week.

Most of the squamous epithelium showed regenerative thickening (RT) in the 10th week from the middle to lower portion of the oesophagus, and basal cell hyperplasia $(\mathrm{BCH})$ was observed from the 20th week. These hyperplastic changes 

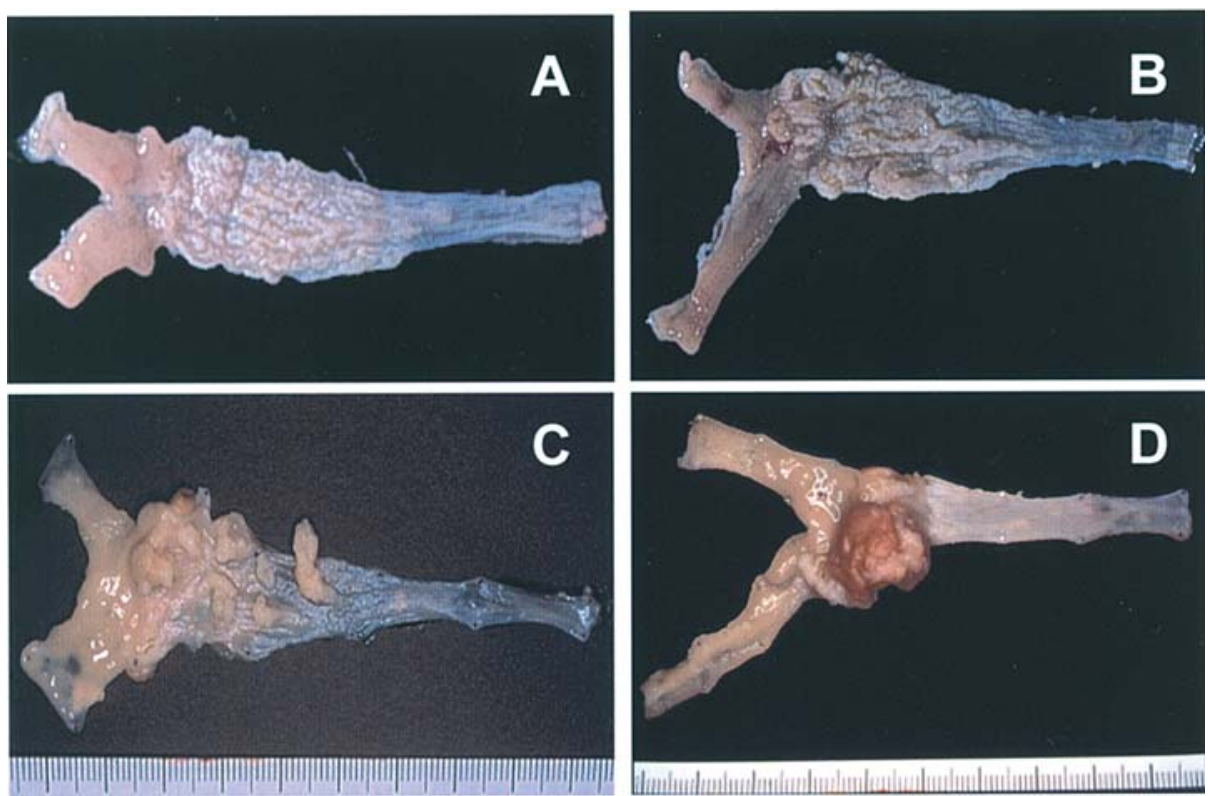

Figure 2. Macroscopic appearance of the resected oesophagus from a rat with duodeno-oesophageal reflux. The mucosa had a glistening surface giving it a cobblestone appearance in the lower portion, and an uneven surface with erosion in the middle portion between the anastomosis site and oral site in the 20th week (A) and 30th week (B). Polypoid adenocarcinoma in the lower oesophagus of a rat with duodeno-oesophageal reflux (C). The tumour had invaded into the liver (D).
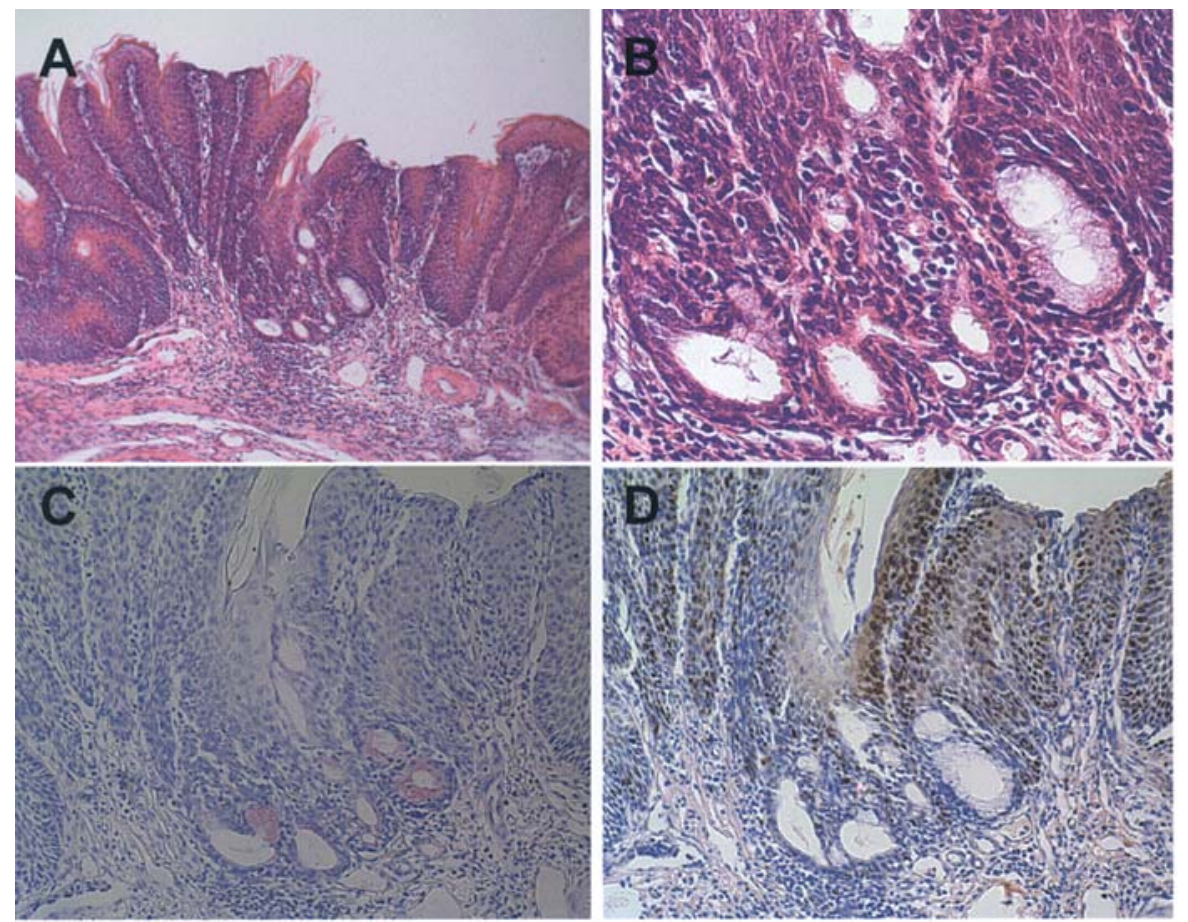

Figure 3. Histological findings in the 20th week after surgery. (A and B), Metaplasia of the gastric foveolar epithelium was observed in the basal layer of the squamous epithelium [original magnifications: HE, (A), x40; (B), x100]. (C), The region of the gastric foveolar epithelial metaplasia was GOS-positive (original magnification HE, x50). (D), The oesophageal basal layer in the region of BCH was positive for PCNA (original magnification: PCNA, x50).

with erosions spread orally from the region at the stoma. PCNA-labelled cells were located in the basal cell layer of the normal squamous epithelium, as well as that showing regenerative thickening and $\mathrm{BCH}$.

In the 20th week, glandular structures were observed scattered throughout the basal layer of the oesophageal squamous epithelium in all animals (Fig. 3A and B). These glandular tissues were comprised of CLE cells, which were not rich in mucins. These buds were constructed from cells with GOS-positive, Con A-negative and HID-AB-negative cytoplasm, similar to the foveolar epithelium of the stomach (Fig. 3C). These columnar epithelia were seen under the proliferative zone (Fig. 3D).

In the 30th week, $\mathrm{BCH}$ and erosion were admixed within the region from the anastomosis toward the oral site. In some of these squamous epithelia, the gland ducts advanced deep 

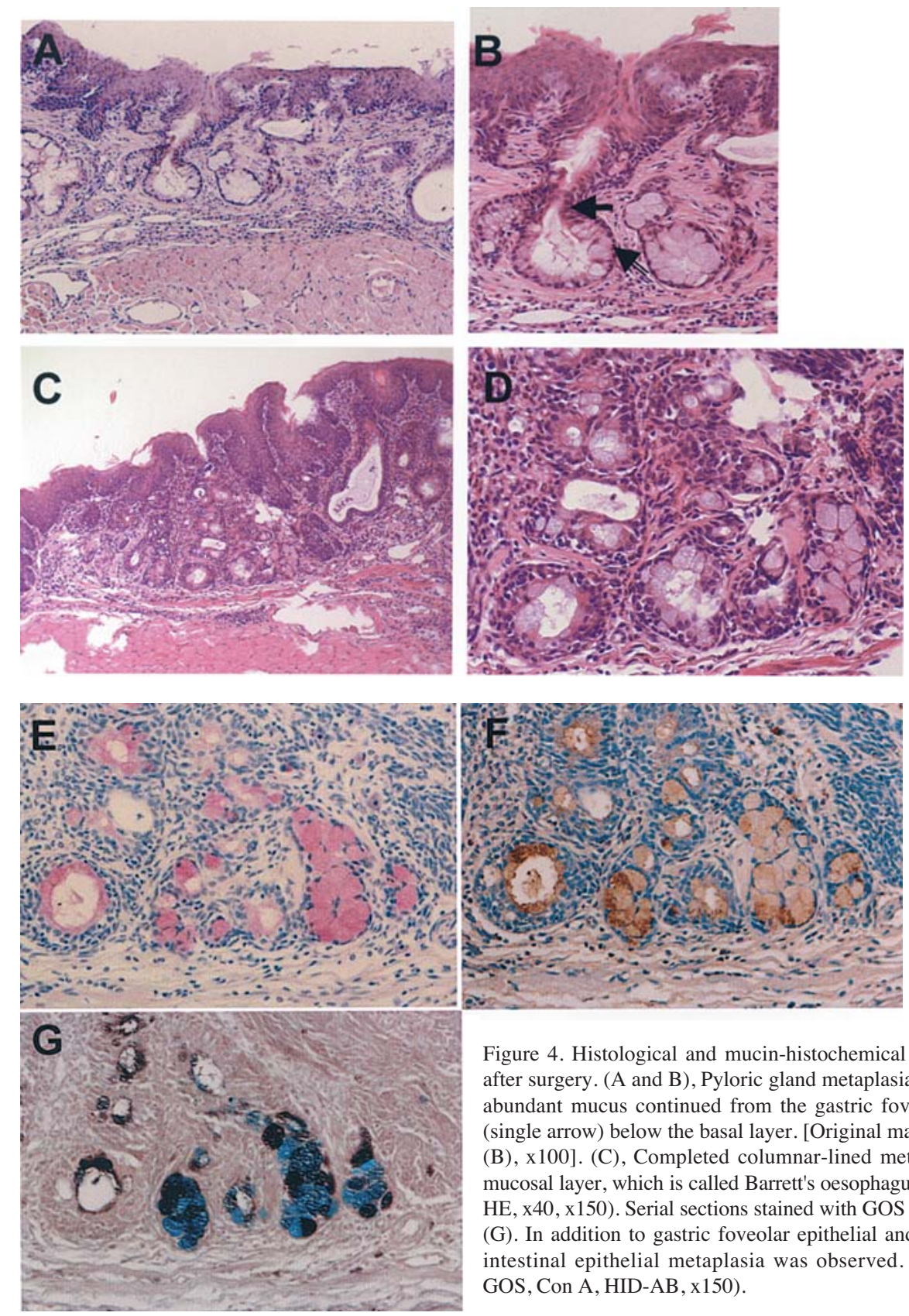

Figure 4. Histological and mucin-histochemical findings in the 30th week after surgery. (A and B), Pyloric gland metaplasia (double arrow) containing abundant mucus continued from the gastric foveolar epithelial metaplasia (single arrow) below the basal layer. [Original magnifications: HE, (A), x40; (B), x100]. (C), Completed columnar-lined metaplasia covered the entire mucosal layer, which is called Barrett's oesophagus. (Original magnifications: HE, $x 40, x 150$ ). Serial sections stained with GOS (E), Con A (F), or HID-AB (G). In addition to gastric foveolar epithelial and pyloric gland metaplasia, intestinal epithelial metaplasia was observed. (Original magnifications: GOS, Con A, HID-AB, x150).

within the mucosa and formed cysts, and metaplasia of the gastric pyloric glands was observed (Fig. 4A and B), in which the cells at the leading region showed increased mucin levels and became Con A-positive. The gland tissue also advanced to the luminal side of the digestive tract and occupied the whole oesophageal mucosal layer, replacing the squamous epithelium (Fig. 4C and D). These phenomena were observed in 11 of 12 animals (92\%). Goblet cells positive for HID-AB, as well as GOS and Con A, were observed in complete columnarlined metaplasia, corresponding to the specialised columnar epithelium of BE in humans, suggesting metaplasia of the intestinal epithelium (Fig. 4E-G). These areas of columnar metaplasia appeared multifocally and sporadically in the lower oesophagus. PCNA-labelled cells were rare in pyloric metaplasia, whereas proliferative cells were seen in foveolar metaplasia extending continuously upward from these regions of pyloric metaplasia.
Specialised columnar epithelium was detected in all animals from the 40th week after surgery. ADC was observed in 5 of $11(45 \%)$ and in 7 of 16 rats (44\%) in the 40th and 50th week after surgery, respectively (Fig. 5A and B). These ADCs arose from the vicinity of the anastomosis and were surrounded by specialised columnar epithelium. On the other hand, the incidences of SCC (Fig. 5C) and ASC (Fig. 5D) were 9 and $9 \%$, and 13 and $6 \%$ in the 40th and 50th week after surgery, respectively. SCC and ASC always accompanied $\mathrm{BCH}$ and developed proximal to the columnar-lined epithelium (Fig. 6). The histopathological findings are summarised in Table I.

\section{Discussion}

The present study indicated that the histogenesis of BE in a rat model involves metaplasia of the gastric foveolar epithelium from the squamous epithelial basal layer developing 

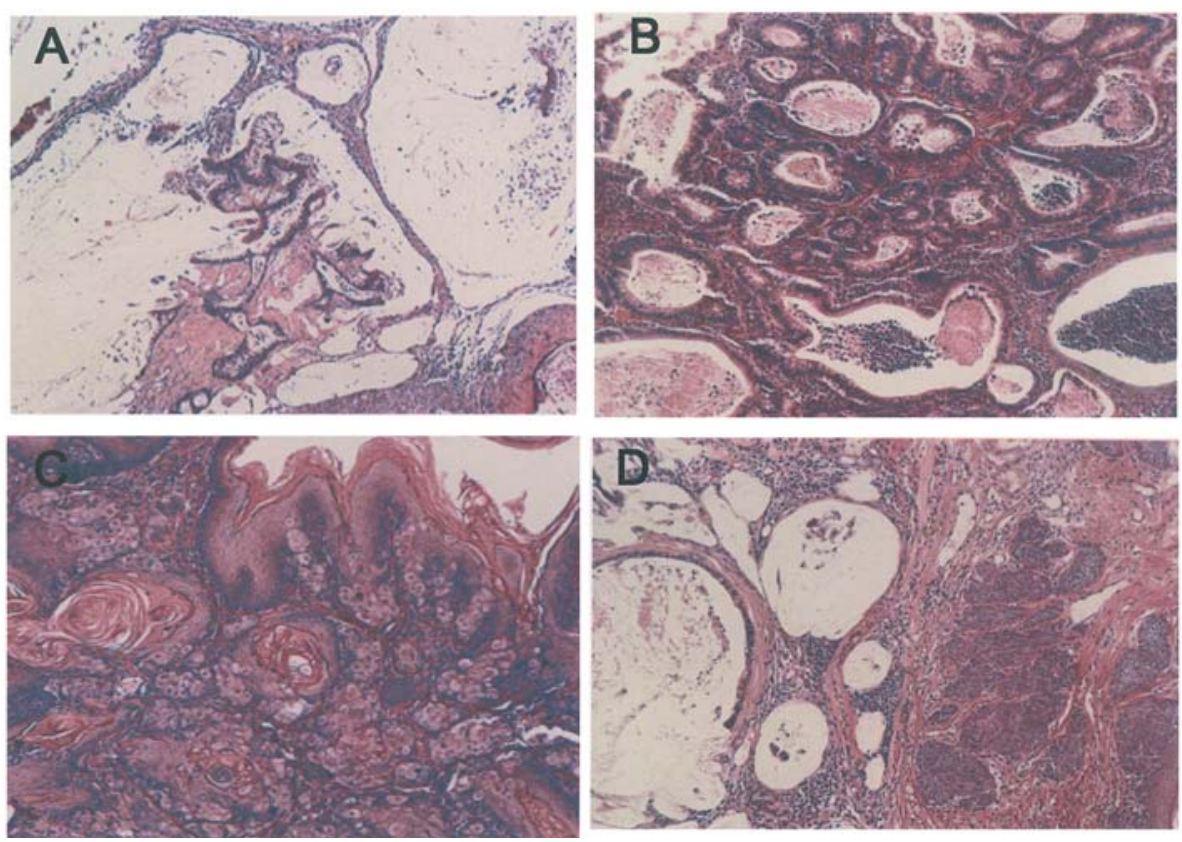

Figure 5. Histological findings of oesophageal carcinoma. (A), Mucin-producing adenocarcinoma. (B), Well-differentiated adenocarcinoma. (C), Squamous cell carcinoma. (D), Adenosquamous carcinoma. [Original magnifications: HE, (A-C), x100; (D), x40].

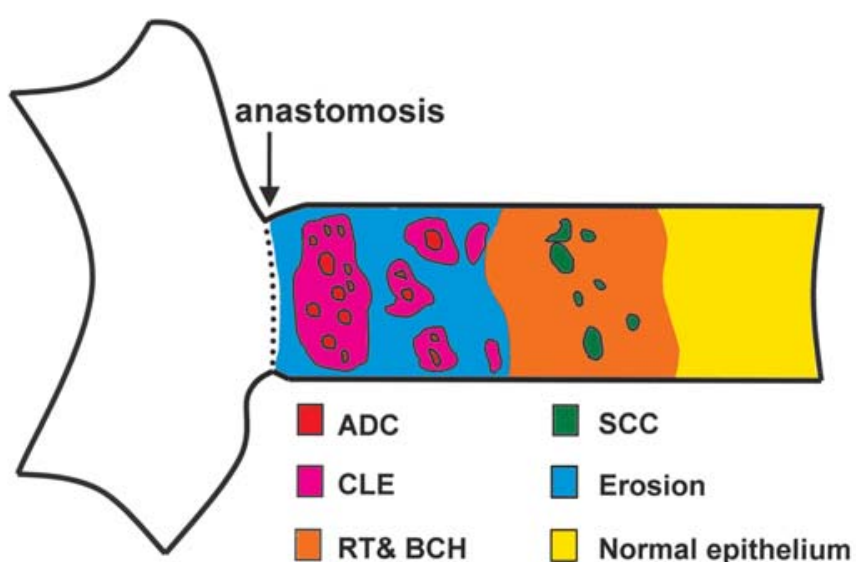

Figure 6. Schematic diagram indicating the relationships between each type of carcinoma and background epithelial patterns in rats with duodenooesophageal reflux. Adenocarcinoma is associated with specialised columnar epithelium, while squamous cell carcinoma and adenosquamous carcinoma arise from proximal squamous oesophagitis.

$\mathrm{BCH}$ due to reflux oesophagitis, followed by induction of 'ulcer-associated cell lineage' (UACL) glands, such as metaplastic gastric pyloric glands and intestinal epithelium. In addition, the ADC had adjacent areas of BE. As rats do not have oesophageal glands proper and as CLE developed in the oesophageal squamous epithelial region, it is suggested that during the repair process, basal cells with pluripotential stem cell qualities, differentiated into columnar cells. These cells stimulated by the duodenal refluxate produced CLE and ADC.

Clinical studies have shown that BE is usually caused by oesophageal reflux of acid and alkali, and that ADC develops from $\mathrm{BE}$ at a high rate $(1,2)$. Patients with complicated $\mathrm{BE}$ refluxate have significantly larger amounts of both acid and duodenal contents as compared to patients with uncomplicated Barrett's oesophagus (5). The duodenal juice contained in the gastric refluxate has been reported to be more injurious to the oesophageal mucosa than gastric juice alone, and it frequently induces BE and ADC (3-5). BE was also generated in totalgastrectomised patients in whom gastric juice was absent (15). Ulcer, constriction, perforation and dysplasia complicating $\mathrm{BE}$ were reported to be related to the presence or absence of duodenal juice contained in the refluxate (16), also suggesting the significance of duodenal juice.

In animal studies, both gastric and duodenal juice reflux have been shown to cause $\mathrm{BE}$, but the frequency was higher with reflux of duodenal juice alone than with reflux of gastric juice alone (8). Kumagai et al reported a duodeno-gastric reflux model with a normal stomach and normal nutritional status with an incidence of BE of $75 \%$ in the 30th and 40th week after surgery (17). In the present study in DER rats, the incidence of BE was $100 \%$ in the 30th and 40th week after surgery. Taken together, these observations suggested that duodenal juice is closely associated with the pathogenesis of BE.

Bremner et al (10) excised the lower oesophageal mucosa and prepared a hiatal hernia to induce reflux in dogs; the deficient mucosa was covered by columnar epithelium in this model, suggesting that the oesophageal columnar epithelium was generated by the creeping repair mechanism of the gastric mucosa. In dogs, Gillen et al (11) confirmed that squamous epithelium remained at the border between the stomach and oesophagus, and the squamous epithelium-deficient region prepared on the upper side was replaced by columnar epithelium. These observations indicated that the replacement cannot be explained by creeping, and they concluded that the columnar epithelium was derived from the oesophageal gland proper. 
Table I. Incidence of oesophageal pathological changes in rats with reflux.

\begin{tabular}{|c|c|c|c|c|c|c|c|c|c|c|}
\hline & \multicolumn{10}{|c|}{ Postoperative week } \\
\hline & \multicolumn{2}{|r|}{10} & \multicolumn{2}{|r|}{20} & \multicolumn{2}{|c|}{30} & \multicolumn{2}{|c|}{40} & \multicolumn{2}{|r|}{50} \\
\hline Regenerative thickening & 12 & $(100)$ & 12 & $(100)$ & 12 & $(100)$ & 11 & $(100)$ & 16 & $(100)$ \\
\hline Basal cell hyperplasia & 8 & $(67)$ & 12 & $(100)$ & 12 & $(100)$ & 11 & $(100)$ & 16 & $(100)$ \\
\hline Erosion & 10 & $(83)$ & 12 & $(100)$ & 12 & $(100)$ & 11 & $(100)$ & 16 & $(100)$ \\
\hline \multicolumn{11}{|l|}{ Columnar-lined epithelium } \\
\hline Foveoral metaplasia & 0 & $(0)$ & 12 & $(100)$ & 12 & $(100)$ & 11 & $(100)$ & 16 & $(100)$ \\
\hline Pyloric metaplasia & 0 & $(0)$ & 1 & (8) & 11 & $(92)$ & 11 & $(100)$ & 16 & $(100)$ \\
\hline $\begin{array}{l}\text { Specialized columnar epithelium } \\
\text { (Intestinal metaplasia) }\end{array}$ & 0 & $(0)$ & 0 & $(0)$ & 11 & $(92)$ & 11 & $(100)$ & 16 & $(100)$ \\
\hline \multicolumn{11}{|l|}{ Carcinoma } \\
\hline Adenocarcinoma & 0 & $(0)$ & 0 & $(0)$ & 0 & (0) & 5 & $(45)$ & 7 & (44) \\
\hline Adenosquamous carcinoma & 0 & $(0)$ & 0 & $(0)$ & 0 & $(0)$ & 1 & (9) & 1 & (6) \\
\hline Squamous cell carcinoma & 0 & $(0)$ & 0 & $(0)$ & 0 & $(0)$ & 1 & (9) & 2 & (13) \\
\hline
\end{tabular}

Data show numbers of rats, with percentages in parentheses.

In the present study, as columnar epithelium was generated in the oesophageal squamous epithelial region at a site distant from the anastomosis and as rats do not have oesophageal glands proper (18), the metaplasia was considered to originate from the stem cells in the squamous epithelial basal layer rather than being formed by creeping of the gastric mucosa. In support of this suggestion, although the entire stomach was excised, the columnar epithelium contained mucus similar to that in the gastric foveolar epithelium and pyloric glands.

BE can be subdivided morphologically into three types: gastric fundic, junctional and specialised columnar types. $\mathrm{Su}$ et al (19) reported that specialised columnar metaplasia and ADC induced in rats with duodeno-gastro-oesophageal reflux were similar to the corresponding lesions in human BE. Thus, the sequential mucus changes in our rat model may reflect the histogenesis of human $\mathrm{BE}$.

The UACL gland has been reported to appear during the process of gastrointestinal mucosal injury repair (20-23). Pyloric gland type cells outgrow from the stem cells and form cysts deep in the mucosa. In the process of mucosal repair in Crohn's disease, Kushima et al (24) reported that proliferating cells in the foveola of the small intestinal mucosa have the potential to differentiate and outgrow as gastric foveolar epithelial type cells or pyloric gland type cells, and then differentiate into goblet cells. Wright (25) observed similar changes in reflux oesophagitis and suggested the involvement of repair/regeneration by UACL.

Therefore, gastric foveolar epithelial type metaplasia occurs in BE, followed by pyloric gland type metaplasia with simultaneous transition to intestinal epithelial metaplasia, such as the UACL gland, completing the formation of Barrett's oesophagus (Fig. 7).

In the present study, there were two histological types of ADC: well-differentiated adenocarcinoma and mucinous

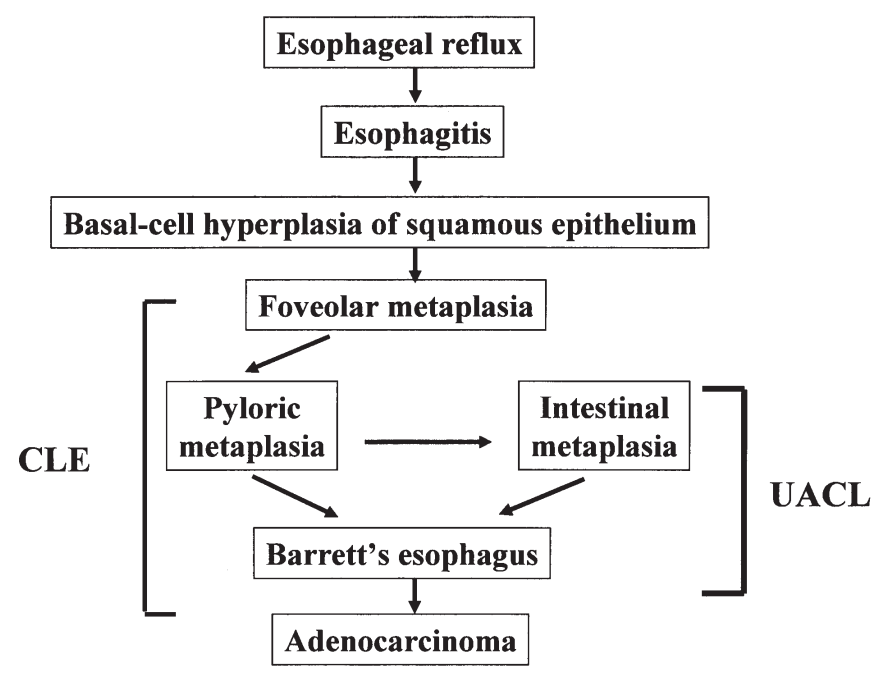

Figure 7. A possible mechanism of the histogenesis of Barrett's oesophagus with ulcer-associated cell lineage and development of adenocarcinoma.

adenocarcinoma. In addition, both SCC and ASC were also detected. Pera et al (26) reported that the carcinomas were not pure ADC but ASC. On the other hand, Goldstein et al (9) detected only ADC with no other types of carcinoma without the use of exogenous carcinogens. In our previous study, approximately $70 \%$ of oesophageal carcinomas were ADC, $17 \%$ were ASC, and $13 \%$ were SCC without treatment with exogenous carcinogens (8), and the incidence rates of the three types of oesophageal carcinoma were similar in the present study. These findings suggest that reflux of duodenal contents induces several types of oesophageal carcinoma.

In conclusion, BE may develop from metaplasia of the stem cells located in the basal layer of the squamous epi- 
thelium due to stimulation by DER. Furthermore, continual stimulation by DER may also lead to ADC from BE.

\section{References}

1. Cameron AJ, Lomboy CT, Pera M, et al: Adenocarcinoma of the esophagogastric junction and Barrett's esophagus. Gastroenterology 109: 1541-1546, 1995

2. Cossentino MJ and Wong RK: Barrett's esophagus and risk of esophageal adenocarcinoma. Semin Gastrointest Dis 14: 128-135, 2003.

3. Champion G, Richter J, Vaezi M, et al: Duodenogastroesophageal reflux: relationship to $\mathrm{pH}$ and importance in Barrett's esophagus. Gastroenterology 107: 747-754, 1994.

4. Kauer W, Peters J, De Meester T, et al: Mixed reflux of gastric and duodenal juices is more harmful to the esophagus than gastric juice alone. Ann Surg 222: 525-533, 1995.

5. Stein HJ, Barlow AP, De Meester TR, et al: Complications of gastroesophageal reflux disease. Role of the lower esophageal sphincter, esophageal acid and acid/alkaline exposure, and duodenogastric reflux. Ann Surg 216: 35-43, 1992.

6. Attwood SE, Smyrk TC, De Meester TR, et al: Duodenoesophageal reflux and the development of esophageal adenocarcinoma in rats. Surgery 111: 503-510, 1992.

7. Miwa K, Segawa M, Takano Y, et al: Induction of oesophageal and forestomach carcinomas in rats by reflux of duodenal contents. Br J Cancer 70: 185-189, 1994.

8. Miwa K, Sahara H, Segawa M, et al: Reflux of duodenal or gastro-duodenal contents induces esophageal carcinoma in rats. Int J Cancer 67: 269-274, 1996.

9. Goldstein SR, Yang GY, Curtis SK, et al: Development of esophageal metaplasia and adenocarcinoma in a rat surgical model without the use of a carcinogen. Carcinogenesis 18: 2265-2270, 1997.

10. Bremner CG, Lynch VP and Ellis FH Jr: Barrett's esophagus: congenital or acquired? An experimental study of esophageal mucosal regeneration in the dog. Surgery 68: 209-216, 1970.

11. Gillen P, Keeling P, Byrne PJ, et al: Experimental columnar metaplasia in the canine oesophagus. Br J Surg 75: 113-115, 1988.

11. Tatematsu M, Ichinose M, Miki K, et al: Gastric and intestinal phenotypic expression of human stomach cancers as revealed by pepsinogen immunohistochemistry and mucin histochemistry. Acta Oathol Jpn 40: 194-504, 1990.

12. Katsuyama $\mathrm{T}$ and Spicer SS: Histochmical differentiation of complex carbohydrates with variants of the concanavalin Ahorseradish peroxidase method. J Histochem Cytochem 26: 233-250, 1978 .

13. Filipe MI: Malignant and inflammatory disease of the gastrointestinal tract. In: Histochemistry in Pathology. Filipe MI and Lake BD (eds). Churchill Livingstone, London, pp126-135, 1983.
14. Ohta T, Yamamoto M, Numata M, et al: Expression of basic fibroblast growth factor and its receptor in human pancreatic carcinomas. Br J Cancer 72: 824-831, 1995.

15. Nishimaki T, Watanabe K, Suzuki T, et al: Early esophageal adenocarcinoma arising in a short segment of Barrett's mucosa after total gastrectomy. Am J Gastroenterol 91: 1856-1857, 1996.

16. Attwood SE, Ball CS, Barlow AP, et al: Role of intragastric and intraoesophageal alkalinisation in the genesis of complications in Barrett's columnar lined lower oesophagus. Gut 34: 11-15, 1993.

17. Kumagai H, Mukaisho K, Sugihara H, et al: Cell kinetic study on histogenesis of Barrett's esophagus using rat reflux model. Scand J Gastroenterol 38: 687-692, 2003.

18. Trskova BL and Meitner ER: Komparative Mikromorphologie der Spiserohre einiger Wirbeltiere. Anat Anz 135: 357-372, 1974.

19. Su Y, Chen X, Klein M, et al: Phenotype of columnar-lined esophagus in rats with esophagogastroduodenal anastomosis: similarity to human Barrett's esophagus. Lab Invest 84: 753-765, 2004.

20. Wright NA, Pike C and Elia G: Induction of a novel epidermal growth factor-secreting cell lineage by mucosal ulceration in human gastrointestinal stem cells. Nature 343: 82-85, 1990.

21. Wright NA, Poulsom R, Stamp G, et al: Trefoil peptide gene expression in gastrointestinal epithelial cells in inflammatory bowel disease. Scand J Gastroenterol Suppl 193: 76-82, 1992.

22. Hanby AM, Jankowski JA, Elia G, et al: Expression of the trefoil peptides pS2 and human spasmolytic polypeptide (hSP) in Barrett's metaplasia and the native oesophageal epithelium: delineation of epithelial phenotype. J Pathol 173: 213-219, 1994

23. Hanby AM, Pera M, Filipe I, et al: Duodenal content reflux esophagitis in the rat: an animal model for the ulcer-associated cell lineage (UACL)? Am J Pathol 151: 1819-1824, 1997.

24. Kushima R, Borchard F and Hattori T: A new aspect of gastric metaplasia in Crohn's disease: bidirectional (foveolar and pyloric) differentiation in so-called 'pyloric metaplasia' in the ileum (letter). Pathol Int 47: 416-419, 1997.

25. Wright NA: Migration of the ductular elements of gut-associated glands gives clues to the histogenesis of structures associated with responses to acid hypersecretory state: the origins of 'gastric metaplasia' in the duodenum of the specialized mucosa of barrett's esophagus and of pseudopyloric metaplasia. Yale J Biol Med 69: 147-153, 1996.

26. Pera M, Brito MJ, Poulsom R, Riera E, Grande L, Hanby A and Wright NA: Duodenal-content reflux esophagitis induces the development of glandular metaplasia and adenosquamous carcinoma in rats. Carcinogenesis 21: 1587-1591, 2000. 